\title{
A One-dimensional Dynamic Model of Thin-walled Octagonal Booms Considering the Cross-section Deformation
}

\author{
Ming Deng, Aimin $\mathrm{Ji}^{*}$, Lei Zhang and Hao Wang \\ College of Mechanical and Electrical Engineering, Hohai University, Changzhou 213022, China \\ ${ }^{*}$ Corresponding author
}

Keywords: Thin-walled, One-dimensional dynamic model, Octagonal cross-section, Cross-section deformation.

\begin{abstract}
In current studies for dynamic behaviors of the telescopic boom with thin-walled section in the aerial work platform, the model was simplified by ignoring the deformation of the cross-section. However, as the working length of the boom increases, the section deformation become larger under loading. For more accurate dynamic analysis, a one-dimensional dynamic model is developed for thin-walled octagonal cross-section boom considering the cross-section deformation in this paper. The model was established that the displacement field was formed through the linear superposition of a set of deformation modes. The deformation modes were defined based on the contour deformations of the cross-section, including out-plane and in-plane, resulted from the unit displacements on the discrete nodes. Then the deformation function between adjacent nodes was approximated with Hermite curve interpolation. The Hamilton's principle was used to develop the governing equation, and further establish the corresponding finite element implementation. Finally, Specific example was also given to verify the accuracy in describing dynamic behaviors of thinwalled octagonal booms.
\end{abstract}

\section{Introduction}

The aerial work platform is used to lift people and instruments to a certain height to carry out construction. As the total length of aerial work vehicle's boom increases, the safety and comfort requirements for people on the boom need to be further ensured and improved. For this reason, some scholars have optimized the section of the booms and proposed an octagonal section. The boom with this cross section has high strength-to-mass ratio, simple manufacturing process and better overall rigidity.[1] However, it is difficult to accurately evaluate their performance due to 'thin walls' comparing with the vast cross-sections. To describe the vibration characteristics of the telescopic booms of the aerial work platform, studies have been performed for several decades. Gao et al.[2] simplified the telescopic beam system to a cantilever beam with end-concentration parameters and take boundary conditions and continuity conditions into consideration when using the classical formula of beam vibration. Alexander Pertsch and Oliver Sawodny[3] used the same simplified model and performed dynamic system solution with the help of Hamilton's principle and flexible dynamics theory. Pertsch et al.[4] modeled the slender ladder as a Euler-Bernoulli beam with a tip mass to carry on studys. Ke and Wang[5] examined the dynamic stability of a functionally graded Timoshenko microbeam employing the modified couple stress theory. Then researches on the dynamic behaviors of thin-walled beams with cross-sections attracted the attention of experts and scholars and widely applied in the engineering. Based on a Timoshenko-Vlasov thin-walled theory, Langseth along with Hopperstad[6] investigated the static and dynamic behaviors of square thin-walled aluminum extrusions under axial loading. Ren et al.[7] also investigated the distortion of cantilever beams with box section under in-plane shear strain.

A one-dimensional dynamic model is developed for thin-walled booms with octagonal crosssection to get more accurate dynamic analysis of the boom in this paper. Firstly, the octagonal crosssection is discretized into eight natural nodes and four intermediate nodes. Then the deformation modes are approximated with nodal displacements, which are defined based on the contour deformation through a polymorphic interpolation. These contour deformations were resulted by the 
imposing of unit displacement on each node. Next, with the linear superposition of a set of deformation modes, the three-dimensional displacement field is reduced to one-dimensional. With the Hamilton's principle, the governing equation is derived. Finally, through the further interpolation of the governing equation, the finite element implementation is obtained. The especial example is also presented to validate the one-dimensional dynamic model.

\section{Cross-section Analysis}

The telescopic boom of the aerial work platform with octagonal cross-section is shown in Fig. 1. Extracting the middle side of the boom, then, the displacement of a point on the midline of the crosssection is defined with the axial $u$, tangential $v$ and normal $w$ components, which are described as positive, along the axis of the natural coordinate system $(n, s, z)$.
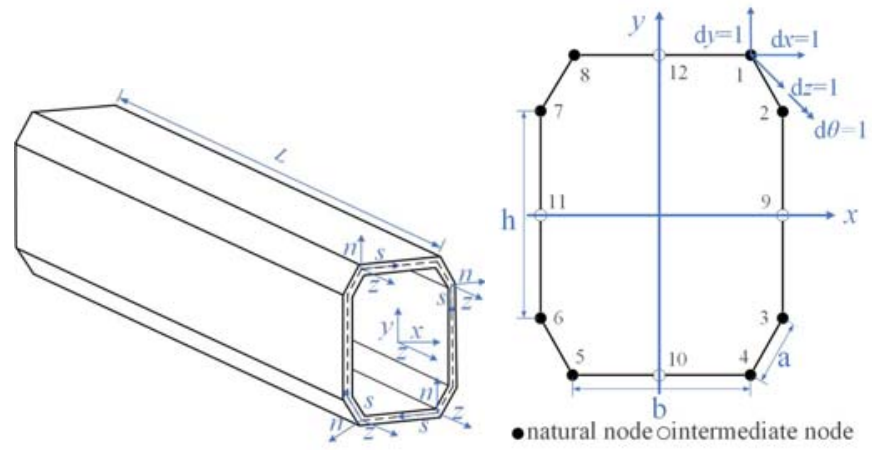

Figure 1. Global coordinate system $(x, y, z)$ and natural coordinate system $(n, s, z)$ and discretization of the crosssections

The global coordinate system $(x, y, z)$ is also shown. The cross-section is divided into eight adjacent walls connected by 8 natural nodes like nodes 1 and 6 . As the length of $b$ and $h$ are much longer than $a, b$ and h are both divided into two segments by introducing intermediate nodes like nodes 9 and 10 , which contribute to describe cross-section deformation.

\section{Displacement Field}

We can find a total of 12 nodes leading to 48 deformation modes in Fig. 2. The displacement field of the cross-section is described by the linear superimposed of a set of basic functions. Each basis function of them can reproduce one type of contour deformation mode and compose of two parts: node displacement, and interpolation between adjacent nodes. First is derived from the imposing of unit displacement on each discrete node. The second part is approximated with interpolation between adjacent nodes. The two interpolation functions used in this paper consist of a Lagrange function of axial and tangential displacement and a cubic Hermite function of normal components.

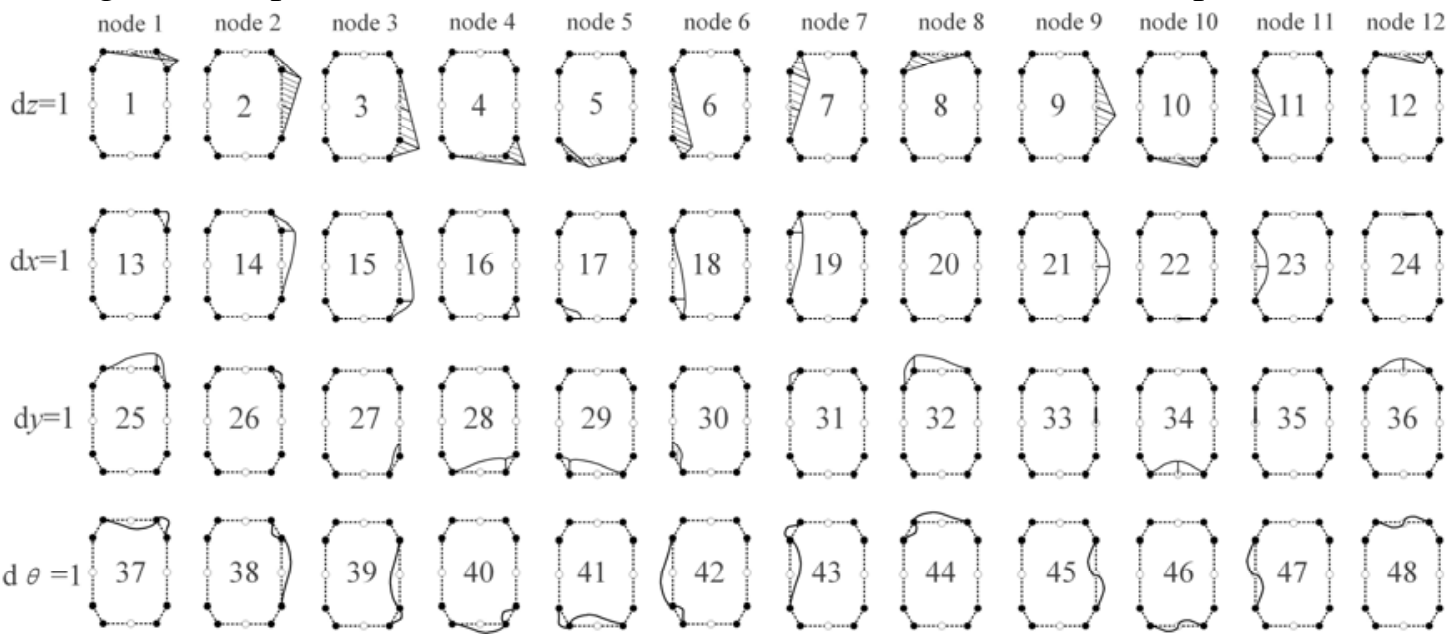

Figure 2. Basis deformation modes of the thin-walled box girder cross-section with 8 discretization nodes 
The deformation modes are all numbered and arranged in the form of matrix with each mode related to the associated node (row) and unit displacement (column). These modes are described with basis functions, which vary along the coordinates for the tangential, normal and axial components.

The displacement field on the cross-section midline, $\boldsymbol{u}=[\boldsymbol{u}(\boldsymbol{s}, \boldsymbol{z}), \boldsymbol{v}(\boldsymbol{s}, \boldsymbol{z}), \boldsymbol{w}(\boldsymbol{s}, \boldsymbol{z})]^{\mathbf{T}}$, is therefore approximated as follow:

$$
\begin{aligned}
& u(s, z)=\boldsymbol{\psi}_{1} \mathbf{x}, v(s, z)=\boldsymbol{\psi}_{2} \mathbf{x}, w(s, z)=\boldsymbol{\psi}_{3} \mathbf{x} \\
& \boldsymbol{\psi}_{1} \mathbf{x}=\left[\varphi_{1}(s), \varphi_{2}(s), \ldots, \varphi_{48}(s)\right] \quad \boldsymbol{\psi}_{2} \mathbf{x}=\left[\psi_{1}(s), \psi_{2}(s), \ldots, \psi_{48}(s)\right] \boldsymbol{\psi}_{3} \mathbf{x}=\left[\omega_{1}(s), \omega_{2}(s), \ldots, \omega_{48}(s)\right]
\end{aligned}
$$

The formulas above correspond to a set of $N$ basis functions. Then the three-dimensional displacement is defined as[8]:

$$
\begin{aligned}
& \boldsymbol{U}=\left[U_{z}(n, s, z), U_{s}(n, s, z), U_{n}(n, s, z)\right]^{\mathrm{T}} \\
& U_{n}(n, s, z)=\boldsymbol{\psi}_{3} \mathbf{x}, U_{s}(n, s, z)=\boldsymbol{\psi}_{2} \mathbf{x}-n\left(\partial \boldsymbol{\psi}_{3} / \partial s\right) \mathbf{x}, U_{z}=\boldsymbol{\psi}_{1} \mathbf{x}-n\left(\partial \boldsymbol{\psi}_{3} / \partial z\right) \mathbf{x}
\end{aligned}
$$

Neglecting structure defect and material uncertainly, the deformation $\varepsilon=\left[\begin{array}{lll}\varepsilon_{z z}(n, s, z) & \varepsilon_{s s}(n, s, z) & \gamma_{z s}(n, s, z)\end{array}\right]^{\mathrm{T}}$ and stress $\sigma=\left[\begin{array}{llll}\sigma_{z z}(n, s, z) & \sigma_{s s}(n, s, z) & \tau_{z s}(n, s, z)\end{array}\right]^{\mathrm{T}}$ are obtained as follows:

$$
\begin{aligned}
& \varepsilon_{z z}=\boldsymbol{\psi}_{1} \partial \mathbf{x} / \partial z-n \boldsymbol{\psi}_{3} \partial^{2} \mathbf{x} / \partial z^{2} \quad \varepsilon_{s s}=\mathbf{x} \partial \boldsymbol{\psi}_{2} / \partial s-n \mathbf{x} \partial^{2} \boldsymbol{\psi}_{3} / \partial s^{2} \gamma_{z s}=\mathbf{x} \partial \boldsymbol{\psi}_{1} / \partial s+\boldsymbol{\psi}_{2} \partial \mathbf{x} / \partial z-2 n\left(\partial \boldsymbol{\psi}_{3} / \partial s\right)(\partial \mathbf{x} / \partial z) \\
& \sigma_{z z}=E^{*} \varepsilon_{z z}+E^{*} v \varepsilon_{s s} \quad \sigma_{s s}=E^{*} v \varepsilon_{z z}+E^{*} \varepsilon_{s s} \quad \tau_{z s}=\mathrm{G} \gamma_{z s} \quad E^{*}=E /\left(1-v^{2}\right) \\
& \boldsymbol{U}=\mathbf{H} \mathbf{x}=\left(\begin{array}{ccc}
\boldsymbol{\psi}_{1} & 0 & -n \partial \boldsymbol{\psi}_{3} / \partial z \\
0 & \boldsymbol{\psi}_{2} & -n \partial \boldsymbol{\psi}_{3} / \partial s \\
0 & 0 & \boldsymbol{\psi}_{3}
\end{array}\right) \mathbf{x} \quad \boldsymbol{\varepsilon}=\mathbf{C U}=\left(\begin{array}{ccc}
\partial / \partial z & 0 & 0 \\
0 & \partial / \partial s & 0 \\
\partial / \partial s & \partial / \partial z & 0
\end{array}\right) \mathbf{U} \quad \boldsymbol{\sigma}=\boldsymbol{E} \boldsymbol{\varepsilon}=\left(\begin{array}{ccc}
E / 1-v^{2} & E v / 1-v^{2} & 0 \\
E v / 1-v^{2} & E / 1-v^{2} & 0 \\
0 & 0 & E / 2(1+v)
\end{array}\right) \boldsymbol{\varepsilon}
\end{aligned}
$$

where $G, E$ and $v$ denote the shear modulus, material Young's modulus and Poisson's ratio.

\section{Governing Equation}

The octagonal boom energy compositions are indispensable for the use of Hamilton's principle, including the strain energy, the potential energy, and the kinetic energy, reading:

$$
U=\frac{1}{2} \iiint_{V} \boldsymbol{\varepsilon}^{T} \boldsymbol{\sigma} d V \quad U_{P}=-\iiint_{V} \boldsymbol{U}^{T} \boldsymbol{p} d A d z \quad T=\frac{1}{2} \iiint_{V} \rho\left(\partial \boldsymbol{U}^{T} / \partial t\right)(\partial \boldsymbol{U} / \partial t) d V
$$

where $A, V$ and $L$ are the section area, the volume and the length of the telescopic boom; $\boldsymbol{p}$ and $\rho$ represent material density and the loading vector. To deduce the governing equation, Hamilton's principle is applied as

$$
\delta \int_{t_{1}}^{t_{2}} L_{a} d t=0,\left.\quad \delta \mathbf{x}\right|_{t=t_{1}}=\left.\delta \mathbf{x}\right|_{t=t_{2}}=0
$$

where $\boldsymbol{L}_{\boldsymbol{a}}=\boldsymbol{T}-\boldsymbol{U}-\boldsymbol{U}_{\boldsymbol{P}}$ is the Lagrangian, and $t_{1}$ and $t_{2}$ are the first and last times. Substituting Eq. (3)-(7) into (8) yields the governing equation of the octagonal boom, reading [9]:

$$
\iiint_{V} \delta \mathbf{x}^{T} \mathbf{H}^{T} \eta \mathbf{H} \partial^{2} \mathbf{x} / \partial t^{2} d A d z+\iiint_{V} \delta \mathbf{x}^{T} \mathbf{H}^{T} \mathbf{c}^{T} \mathbf{E} \mathbf{c h} \mathbf{x} d A d z-\iiint_{V} \delta \mathbf{x}^{T} \mathbf{H}^{T} \mathbf{p} d A d z=0
$$

\section{Finite Element Implementation}

The quadratic functions are used to simulation the displacement with the second-order partial derivative in Eq. (8).

$$
\mathbf{x}=\mathbf{N} \mathbf{x}=\left[\begin{array}{lll}
\mathbf{N}_{1} & \mathbf{N}_{2} & \mathbf{N}_{3}
\end{array}\right] \mathbf{x}, \mathbf{N}_{1}=\left(2 z^{2} / l^{2}-3 z / l+1\right) \mathbf{I}_{48}, \mathbf{N}_{2}=\left(4 z / l-4 z^{2} / l^{2}\right) \mathbf{I}_{48}, \quad \mathbf{N}_{3}=\left(2 z^{2} / l^{2}-z / l+1\right) \mathbf{I}_{48}
$$


where $\mathbf{X}$ and $\mathbf{N}$ are the vector of nodal displacement and the matrix shape function; $l$ is the length of each element.

Substituting Eq. (10) into (9) leads to the finite element formulation

$$
\begin{aligned}
& \mathbf{m} \partial^{2} \mathbf{x} / \partial t^{2}+\mathbf{k x}=\mathbf{f} \\
& \mathbf{m}=\int_{L} \int_{A} \mathbf{N}^{T} \mathbf{H}^{T} \eta \mathbf{H} d A d z, \mathbf{k}=\int_{L} \int_{A} \mathbf{N}^{T} \mathbf{H}^{T} \mathbf{c}^{T} \mathbf{E} \mathbf{c H} d A d z, \mathbf{f}=\int_{L} \int_{A} \mathbf{N}^{T} \mathbf{H}^{T} \mathbf{p} d A d z
\end{aligned}
$$

where $\mathbf{m}, \mathbf{k}$ and $\mathbf{f}$ are the mass matrix, the stiffness matrix and the element force matrix respectively.

\section{Numerical Examples}

To validate the model, numerical examples are taken into account. Considering one boom, specific parameters are set as $L=4.2 \mathrm{~m}, h=0.34 \mathrm{~m}, b=0.28 \mathrm{~m}, a=0.1 \mathrm{~m}, t=0.03 \mathrm{~m}, E=200 \mathrm{GPa}, v=0.3$, $\rho=7830 \mathrm{Kg} / \mathrm{m} 3$.

\section{Case 1: A Fixed-free End Condition}

After checking of convergence of the proposed finite element, it is used to model a fixed-free boom for free vibration analysis considering actual working conditions of aerial work platform.

Table 1. Comparison of The First 8 Natural Frequencies of Cantilever Octagonal Boom

\begin{tabular}{|c|c|c|c|}
\hline Mode & Present mode [Hz] & ANSYS shell [Hz] & Relative errors [\%] \\
\hline 1st & 24.244 & 24.221 & $0.09 \%$ \\
\hline 2nd & 30.407 & 30.349 & $0.19 \%$ \\
\hline 3rd & 133.480 & 133.740 & $-0.19 \%$ \\
\hline 4th & 159.270 & 166.510 & $-4.34 \%$ \\
\hline 5th & 166.470 & 167.910 & $-0.85 \%$ \\
\hline 6th & 172.490 & 172.390 & $0.06 \%$ \\
\hline 7th & 301.030 & 301.210 & $-0.06 \%$ \\
\hline 8th & 307.890 & 312.800 & $1.56 \%$ \\
\hline
\end{tabular}

As shown in the Table 1, the first 8 natural frequencies obtained from the model are compared with the results from ANSYS shell theory. The first 8 natural frequencies of the cantilevered octagonal booms model are calculated with 48 over the cross-section(A) and 60 elements along the length(L), while the ANSYS model is directly discretized into 1080 Shell 181 elements. We can find that the relative errors of natural frequencies between the present model and ANSYS shell theory is controlled within $5 \%$.

To further validate the versatility, Fig. 3 indicates the comparison of the modal shapes from $1^{\text {th }}$ to $8^{\text {th }}$ to show the accuracy of describing three-dimensional behaviors.
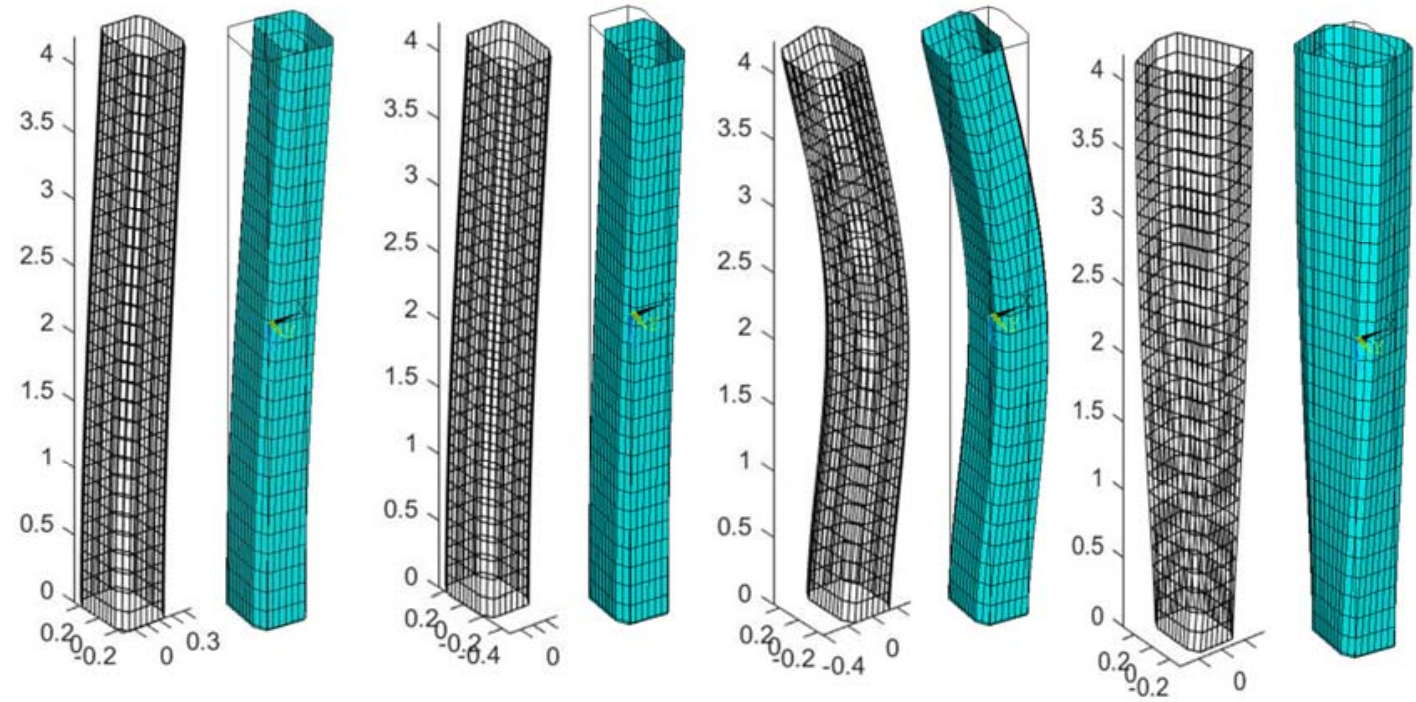

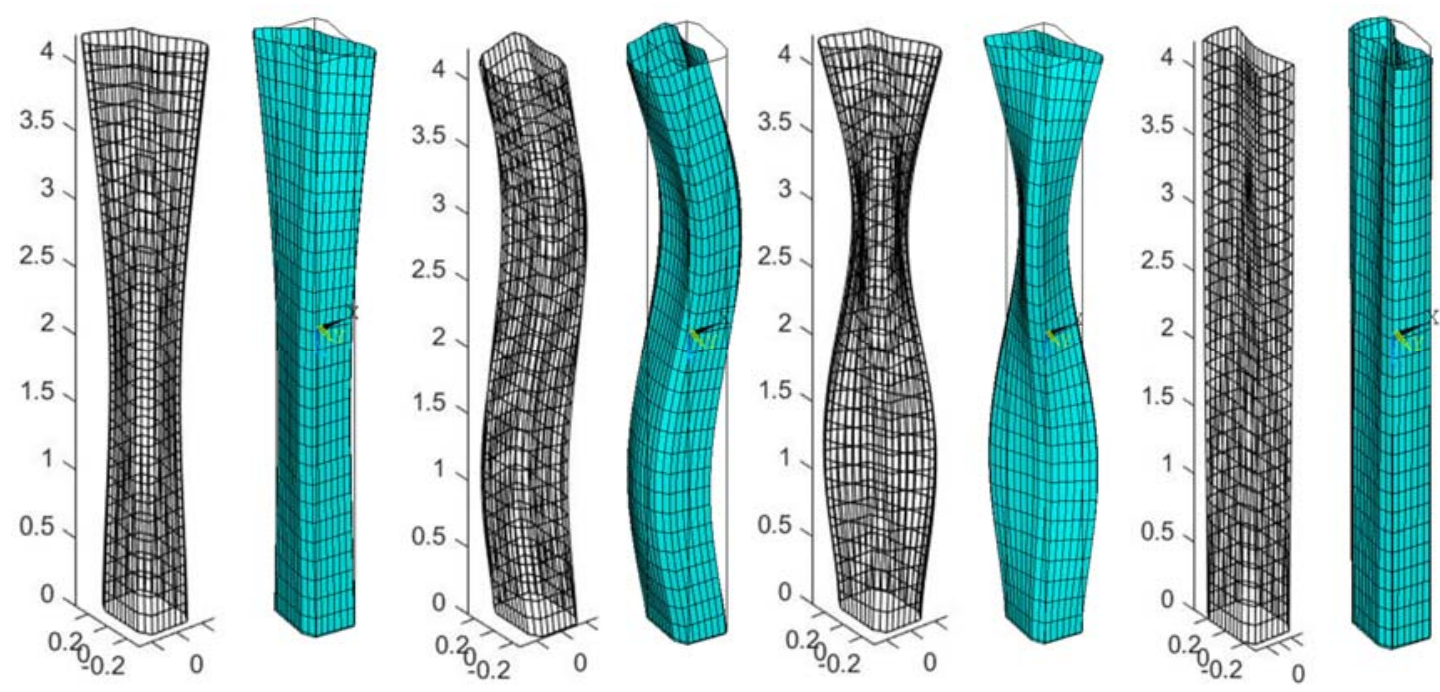

Figure 3. Comparison of modal shapes (the light ones) of the cantilevered octagonal booms with ANSYS shell model.

\section{Case 2: Simplification of the Model}

Fig. 2 shows that 48 modes are employed to construct a one-dimensional model of the octagonal booms. This may lead our research to be cumbersome and ineffective. To simply the model and reduce the number of the generalized coordinates, the absolute values of the amplitudes of the modes shape from $1^{\text {st }}$ to $8^{\text {th }}$ are shown in Fig. 4. From these eight figures we can find only the generalized coordinates of $13^{\text {th }}$ to $20^{\text {th }}, 25^{\text {th }}$ to $32^{\text {th }}$ and $37^{\text {th }}$ to $44^{\text {th }}$ contribute to the change of the boom's mode shapes. It is worth mentioning that these 32 generalized coordinates are caused by the application of unit displacement to the natural nodes of the in-plane, including the tangential $(v)$, normal $(w)$ and rotation $(\theta)$ components. Thus, it is reasonable to obtain a reduced set of generalized coordinates without affecting the accuracy of reproducing the three-dimensional deformation of thin-walled octagonal booms.
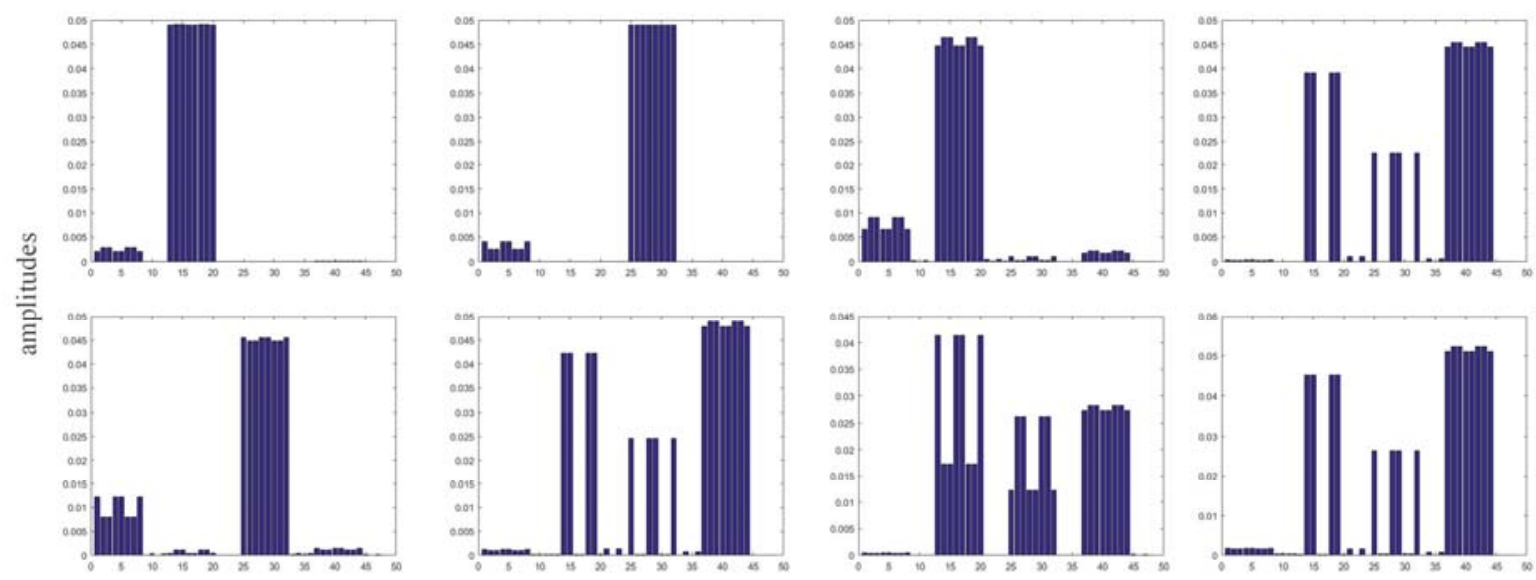

mode numbers

Figure 4. Amplitudes of basis deformations of the first to eighth modals of the cantilevered thin-walled octagonal booms

\section{Summary}

In this paper, the deformation of the cross-section is taken into consideration to improve the accuracy of describing the vibration behaviors of aerial work platform. In the process, the cross-section is splited to the intermediate and natural nodes. Then section deformation modes are considered to approximate interpolations of nodal displacements through a polymorphic interpolation. Then the octagonal boom displacement field is approximated by the linear superposition of deformation modes. Applying Hamilton's principle, the governing differential equation is derived, and then interpolated 
to establish the corresponding finite element implementation. Specific examples showed that the present model is consistent with three-dimensional models with less computation and is of better accessibility. Besides, the examples also show that different generalized coordinates vary greatly in participating the structure vibration mode. Thus, furthermore studies will be invested in the future to reduce the number of generalized coordinates but without affecting the accuracy of the onedimensional model.

\section{Acknowledgement}

This paper was financially supported by National Science Foundation of China (Grant No.51805144), Natural Science Foundation of Jiangsu Province (Grant No. BK20170300), and the Foundation of Changzhou Key Laboratory of Aerial Work Equipment and Intelligent Technology.

\section{References}

[1] Sharafi P., Teh L. H., Hadi M. N. S. Shape optimization of thin-walled steel sections using graph theory and ACO algorithm. Journal of Constructional Steel Research, Vol. 101, 2014, p. 331-341.

[2] L.C. GAO, R.M. TENG, X. WANG, Vibration behaviors of the boom system of a telescopic boom aerial work platform[J]. Journal of vibration and shock, 2016, 35(10): 225-230.

[3] Zimmert N, Pertsch A, Sawodny O. 2-DOF Control of a Fire-Rescue Turntable Ladder[J]. IEEE Transactions on Control Systems Technology, 2012, 20(2): 438-452.

[4] Pertsch, A., Zimmert, N., Sawodny, O.. Modeling a fire-rescue turntable ladder as piecewise Euler-Bernoulli beam with a tip mass[P]. Decision and Control, 2009 held jointly with the 2009 28th Chinese Control Conference. CDC/CCC 2009. Proceedings of the 48th IEEE Conference on, 2009:7321-7326.

[5] Ke L-L, Wang Y-S. Size effect on dynamic stability of functionally graded microbeams based on a modified couple stress theory. Compos Struct 2011;93(2):342e50.

[6] Langseth M., Hopperstad O. S. Static and dynamic axial crushing of square thin-walled aluminum extrusions. International Journal of Impact Engineering, Vol. 18, Issue 7, 1996, p. 949-968.

[7] Y. Ren, W. Cheng, Y. Wang, B. Wang, Analysis of the distortion of cantilever box girder with inner flexible diaphragms using initial parameter method, Thin. Wall. Struct. 117 (2017) 140-154.

[8] Garcea, G.; Gonçalves, R.; Bilotta, A. et al. Deformation modes of thin-walled members: A comparison between the method of Generalized Eigenvectors and Generalized Beam Theory. ThinWalled Struct. 2016, 100, 192-212

[9] Zhu, Z.; Zhang, L.; Shen, G.; Cao, G. A one-dimensional higher-order theory with cubic distortional modes for static and dynamic analyses of thin-walled structures with rectangular hollow sections. Acta Mech. 2016,227, 2451-2475 